\title{
Trabeculotomy in pseudoexfoliation of the lens capsule
}

\author{
W. E. GILLIES \\ From the Glaucoma Unit, Royal Victorian Eye and Ear Hospital, Melbourne, Australia
}

SUMMARY Trabeculotomy was performed on 12 eyes of 11 patients with glaucoma associated with pseudoexfoliation of the lens capsule. Pressure was lowered in all eyes, in 10 eyes to $15 \mathrm{mmHg}$ or less, 7 with no medication but 3 with medication. A subconjunctival bleb was present in 5 patients but was not necessary for a good result. These results suggest that the raised intraocular pressure which often occurs in pseudoexfoliation of the lens capsule is due to interference with flow of aqueous across the trabecular meshwork. Trabeculotomy seems a safe and effective operation in glaucoma associated with pseudoexfoliation of the lens capsule.

Pseudoexfoliation of the lens capsule is a condition in which there is a high incidence of glaucoma. There is also a deposition of pseudoexfoliative material and pigment in the trabecular tissue at the angle of the anterior chamber. Does the deposition of this material cause an increase in resistance to the flow of aqueous into the canal of Schlemm with a rise in the intraocular pressure? If it does, then the surgical operation of trabeculotomy, which divides the trabecular tissue between the anterior chamber and the canal of Schlemm, may lower the intraocular pressure. This paper reviews a group of patients with glaucoma and pseudoexfoliation of the lens capsule on whom trabeculotomy was performed.

\section{Material and methods}

The surgical operation of trabeculotomy was performed on 12 eyes of 11 patients all with glaucoma associated with pseudoexfoliation of the lens capsule. The operation was performed on eyes with progressive field loss in the presence of an intraocular pressure which could not be controlled to less than $20 \mathrm{mmHg}$ by full medical therapy. Patients were followed up for periods of 6 months to $3 \frac{1}{2}$ years postoperatively.

Trabeculotomy was performed after the method of Harms and Dannheim (1969), and Dannheim (1972). The conjunctiva was reflected. A half thickness scleral flap $3 \times 3 \mathrm{~mm}$ based on the limbus

Address for reprints: W. E. Gillies, FRCS, Glaucoma Unit, Royal Victorian Eye and Ear Hospital, Melbourne 3000, Australia was raised and a linear incision made down to the canal of Schlemm. Trabeculotomy probes were then passed for about $60^{\circ}$ along the canal of Schlemm to either side and rotated into the anterior chamber. The scleral flap was sutured back in place with 7 or more 10.0 nylon sutures and the conjunctiva was closed. The anterior chamber was not lost throughout the procedure, and the wound was watertight at the conclusion of surgery.

\section{Results}

The intraocular pressure was lowered in all the eyes (Table 1). In 10 eyes the pressure was lowered to $15 \mathrm{mmHg}$ or less, in 7 without any treatment and in 3 with the help of medical treatment. Of the 7 eyes with a pressure of $15 \mathrm{mmHg}$ or less with no treatment 4 had a subconjunctival drainage bleb and 3 did not. One other eye with a bleb needed miotic drops to attain a pressure of $20 \mathrm{mmHg}$.

In all patients gonioscopy showed a cleft present in the region of the canal of Schlemm. This cleft seldom extended over the full extent of the trabeculotomy and tended to extend forwards towards Schwalbe's line as if the trabecular tissue had disinserted from this line. Reflux of blood from the ruptured canal of Schlemm into the anterior chamber was seen gonioscopically in 6 of the 12 eyes; in 2 of these eyes miotic drops were necessary to get a satisfactory intraocular pressure.

There were no serious complications in any of the eyes. A small hyphaema was common postoperatively but cleared before the patients went home in 5 days. Some peeling of Descemet's 
Table 1 Results of trabeculotomy in glaucoma with pseudoexfoliation of lens capsule

\begin{tabular}{|c|c|c|c|c|c|c|c|c|}
\hline \multirow{2}{*}{ Patient } & \multirow{2}{*}{$\operatorname{Sex}$} & \multirow{2}{*}{ Age } & \multirow{2}{*}{$\begin{array}{l}\text { Follow-up } \\
\text { (months) }\end{array}$} & \multirow{2}{*}{$\begin{array}{l}\begin{array}{l}\text { Pressure } \\
\text { Preop }\end{array} \\
\text { No } R_{x}\end{array}$} & $\begin{array}{l}\text { Pressure } \\
\text { Preop }\end{array}$ & \multirow{2}{*}{$\begin{array}{l}\text { Pressure } \\
\text { postop }\end{array}$} & \multirow{2}{*}{ Bleb } & \multirow{2}{*}{$\begin{array}{l}\text { Reflux of } \\
\text { blood }\end{array}$} \\
\hline & & & & & $O n R_{x}$ & & & \\
\hline 1 & $\mathbf{F}$ & 76 & 42 & 28 & 26 & $15\left(R_{x}\right)$ & No & - \\
\hline 2 & $\mathbf{M}$ & 67 & 37 & 43 & 30 & 12 & No & - \\
\hline 3 & $\mathbf{F}$ & 56 & 37 & 50 & 28 & 11 & Yes & - \\
\hline 4 & $\mathbf{M}$ & 73 & 37 & 37 & 27 & 9 & Yes & + \\
\hline \multirow[t]{2}{*}{6} & M (R) & 65 & 23 & 43 & 20 & 15 & Yes & + \\
\hline & (L) & & 23 & 43 & 34 & $20\left(R_{x}\right)$ & Yes & -1 \\
\hline 7 & F & 59 & 22 & 43 & 26 & 14 & No & - \\
\hline 8 & $\mathbf{F}$ & 71 & 18 & 35 & 25 & $12\left(R_{x}\right)$ & No & - \\
\hline 9 & $\mathbf{M}$ & 70 & 13 & 58 & 28 & 16 & No & - \\
\hline 10 & $\mathbf{F}$ & 78 & 8 & 40 & 26 & 14 & Yes & + \\
\hline 11 & $\mathbf{M}$ & 71 & 6 & 32 & 25 & $11\left(R_{x}\right)$ & No & + \\
\hline
\end{tabular}

membrane in the region of the operation was common but caused no ill effects. There were no flat or shallow anterior chambers postoperatively. The anterior chamber was not lost at operation in any case. In 2 eyes the pressure has become more difficult to control with lapse of time since the operation.

\section{Comment}

Trabeculotomy is a safe and effective operation for glaucoma associated with pseudoexfoliation of the lens capsule. First described by Burian (1960) and Smith (1960), it attempts to remove the resistance to aqueous flow across the trabecular meshwork. Its success in this type of glaucoma suggests that the deposition of pseudoexfoliative material and pigment in the trabecular meshwork causes a rise in the trabecular resistance to aqueous flow, with a consequent rise in intraocular pressure.

Whether the deposition of pseudoexfoliative material and pigment causes a rise in intraocular pressure in a particular eye probably depends on the pre-existing state of the trabecular meshwork. Patients with a very low resistance to aqueous outflow are unlikely to develop a rise in intraocular pressure, while those with a marginal resistance are more likely to do so. As resistance to aqueous outflow may vary between two eyes in a patient, it occasionally happens that one eye with clinical pseudoexfoliation will have a normal intraocular pressure while the other with no pseudoexfoliation will have a raised intraocular pressure.

The presence of a subconjunctival bleb was not necessary for a successful result, though it was common among eyes with a good result. Although great care was taken to secure watertight scleral closure, it seems inevitable that subconjunctival leakage of aqueous will occur in some eyes once trabecular resistance is removed. The presence of a reflux of blood from the trabeculotomy site in 6 eyes is strong evidence that a communication has been established between the canal of Schlemm and the anterior chamber. As blood filling of the canal of Schlemm does not always occur at gonioscopy, blood reflux from the region of the trabeculotomy cannot be expected in every eye, even if the trabeculotomy is patent. Not all patients with reflux of blood from the trabeculotomy site achieved a low intraocular pressure without miotic drops.

The use of miotic drops lowered the intraocular pressure if it was still raised, though miotic drops are commonly thought to act by the way of the pull of the scleral spur on the trabecular tissue. Possibly they act after trabeculotomy by opening the trabeculotomy cleft.

\section{References}

Burian, H. M. (1960). A case of Marfan's syndrome with bilateral glaucoma. American Journal of Ophthalmology, 50, 1187-1192.

Dannheim, R. (1972). Trabeculotomy. Transactions of the American Academy of Ophthalmology and Otolaryngology, 76, 375.

Harms, H., and Dannheim, R. (1969). Epicritical consideration of 300 cases of trabeculotomy ab externo. Transactions of the Ophthalmological Societies of the United Kingdom, 89, 491.

Smith, R. (1960). A new technique for opening the canal of Schlemm. British Journal of Ophthalmology, 44, 370. 